\title{
Zinkoxyd und Chlorophyll als optische Sensibilisatoren
}

\section{Doctoral Thesis}

Author(s):

Böhi, Jakob

Publication date:

1929

Permanent link:

https://doi.org/10.3929/ethz-a-000092389

Rights / license:

In Copyright - Non-Commercial Use Permitted

Originally published in:

Sammelband ETH-BIB 


\title{
Zinkoxyd und Chlorophyll als optische Sensibilisatoren
}

\author{
Von der
}

Eidgenössischen Technischen Hochschule in Zürich

zur Erlangung der

Würde eines Doktors der technischen Wissenschaften

\author{
genehmigte \\ Promotionsarbeit \\ vorgelegt von \\ Jakob Böhi, Dipl. Ingenieur-Chemiker \\ aus Schönholzerswilen (Thurgau) \\ Referent: Herr Prof. Dr. E. Baur \\ Korreferent: Herr Prof. Dr. W. D. Treadwell
}

Nr. 561

BASEL

Buchdruckerei Emil Birkhäuser \& Co.

1929 
stoffes wieder unmöglich war. Immerhin fand ich eine Reihe von Farbstoffen, die sich eigneten.

Im binären System und auch mit Terpentinöl als Depolarisator konnte ich in keinem Falle eine Photolyse beobachten.

Lanacylblau, ein Farbstoff, der in methylalkoholischer Lösung schon in 2 Stunden bei Chlorophyllgegenwart ausbleichte, blieb hier bei Belichtung ganz unverändert.

Erst bei Anwendung von Phenylhydrazin als. Depolarisator konnte ich eine durch Licht und Chlorophyll bewirkte Reduktion einiger Farbstoffe beobachten.

Bei Anwendung der Lösungsmittel Benzol und Wasser bleichten im Licht bei Gegenwart von Chlorophyll und Phenylhydrazin die Farbstoffe: Echtrot A V, Lanacylblau und Diaminrosa aus. Bei Verwendung von Tetrachlorkohlenst off und Wasser, Echtrot A V, Lanacylblau und Orange $R$, und bei Verwendung von Terpentinöl und Wasser: Echtrot A V.

Die Sensibilisierung der Photolysen geht also in diesen zweiphasigen Systemen bedeutend schwerer vor sich, als in monomolekularer Lösung.

Der Hauptgrund dürfte wohl darin zu suchen sein, dass die wirksame Oberfläche der Chlorophylls in der gewählten Anordnung (makroskopische Emulsion) viel zu klein ist.

\section{Zusammenfassung.}

1. Es wird der quantitative Verlauf der durch Zinkoxyd sensibilisierten Photolyse von Berlinerblau im binären und ternären System verfolgt.

2. Eine wässerige Kupfersalzlösung wird durch Anwesenheit von Zinkoxyd lichtempfindlich.

Bei Anwesenheit von anodischen Depolarisatoren wird das Cupri-ion über die Cuprostufe zu metallischem Kupfer reduziert. Im binären System bildet sich Kupferoxyd.

3. Zinkoxyd bewirkt in Licht die Vereinigung von Knallgas zu Wasser und Hydroperoxyd.

4. Chlorophyll in methylalkoholischer Lösung bringt die photolytische Reduktion zahlreicher Farbstoffe, die den verschiedensten Farbstoffklassen angehören, hervor.

Diese Arbeit wurde im Physikalisch-chemischen Institut der Eidgen. Techn. Hochschule in Zürich unter Leitung von Herrn Prof. Dr. $E$, Baur ausgeführt. Es ist mir eine angenehme Pflicht, meinem hochverehrten Lehrer für die Anregung und die stets wohlwollende Unterstützung dieser Arbeit meinen herzlichsten Dank auszusprechen. 